\title{
A PICTORIAL ESSAY ON RADIOGRAPHY OF SWALLOWING AND ESOPHAGEAL DISORDERS
}

\author{
Damjana KLJUČEVŠEK
}

University Clinical Centre, Children's Hospital Ljubljana, Radiology Unit

\author{
Corresponding author: \\ Damjana Ključevšek \\ University Clinical Centre \\ Children's Hospital Ljubljana \\ Radiology Unit \\ Bohoričeva 20 \\ 1000 Ljubljana, Slovenia \\ damjana.kljucevsek@gmail.com \\ Tel: + 38615229264 \\ Fax.: + 38615222993
}

Received: November 9, 2014

Accepted: December 26, 2014

Copyright (C) 2015 by

University Clinical Centre Tuzla.

E-mail for permission to publish:

paediatricstoday@ukctuzla.ba

\begin{abstract}
Numerous abnormalities, both of congenital and acquired causes, can compromise the swallowing process and the ability of the esophagus to transmit the food from the mouth to the stomach in pediatric population. Patients often present a history of aspiration, dysphagia or feeding difficulties. The imaging evaluation typically begins with a fluoroscopic examination of the swallowing process and esophagus (esophagram), using either barium or water soluble contrast medium. This pictorial essay delineates the imaging features of normal anatomy and physiology of the swallowing and the esophagus, as well as the imaging features of common pediatric swallowing dysfunctions and various esophageal abnormalities. It is designed in order to assist radiologists in providing an appropriate diagnosis when confronted with a suspected disease in this anatomic region, and when appropriate, what additional testing to recommend. It is also designed to inform pediatricians about the wealth of diagnostic information that may be collected when performing an esophagram; a relatively simple radiographic examination. Conclusion - Radiographic imaging of swallowing and the esophagus remains the primary imaging modality in patients with a history of aspiration, dysphagia and feeding problems. It offers valuable diagnostic information related to anatomy, morphology and peristalsis.
\end{abstract}

Key words: Radiography of swallowing - Radiography of esophagus • Children - Swallowing disorders - Esophageal abnormalities.

\section{Introduction}

Esophagram (esophagogram, esophagography) is radiographic contrast imaging of the esophagus. The esophagus is evaluated dynamically with real-time fluoroscopy using orally administered contrast medium (CM). Contrast can be followed through the stomach and duodenum to the ligament of Trietz, to perform an upper gastrointestinal series (UGI). Esophagram maintains an important role in the diagnostic algorithms concerning the esophagus pathology despite newer cross sectional techniques like computer tomography (CT) and magnetic resonance (MR), and the increasing role of endoscopy. Esophagram is simple, quickly performed, low in radiation dose, inexpensive, available in every hospital, and there is no need for sedation. At the same time it gives important and useful anatomical, morphological, and functional information. Normal anatomy and physiology of the esophagus should be well known when evaluating the esophagus as well as the child's history of feeding and his or her clinical condition (1). All this information helps 
to plan the performance of the esophagram: the type of CM used during examination and eventually the modification of the examination protocol. Ultrasound (US), CT, and MR imaging can also be useful in the esophagus evaluation, mostly in the cases of extrinsic esophageal compressions and intramural lesions.

This pictorial essay delineates the radiographic esophageal anatomy, imaging techniques, and common pathology seen in the swallowing dysfunction and in the pediatric esophagus including congenital anomalies, motility disorders, esophageal mucosa and lumen changes (stenosis, outside impressions), and gastroesophageal junction (GEJ) disorders.

\section{Normal anatomy and physiology of the esophagus}

The esophagus is a tubular structure extending from distal hypopharynx at the level of the cricopharyngeus muscle (upper esophageal sphincter) to the GEJ (lower esophageal sphincter). Its main role is to facilitate the passage of food from the mouth to the stomach. Thus, its caliber varies with peristaltic activity. Normal extrinsic impressions on the esophagus include the cricopharyngeal muscle, aortic impression, left main stem bronchus impression, left atrium and the diaphragm (1).

The GEJ represents the so called lower esophageal sphincter (LES) (2). The largest portion is the vestibule, which spans the diaphragmatic hiatus. The upper border of the vestibule is the A ring (muscular contraction at the junction of tubular and vestibular esophagus), while the lower is the B ring (mucosal ring at anatomic squamocolumnar junction - Z-line). These two rings are usually not seen in children.

Changes in the anatomy and physiology of the esophagus occur with growth and de- velopment. Infant anatomy is different from that in older children. The structures of the mouth and pharynx are close together, the larynx is higher and smaller, which facilitates safe swallowing and might mask a significant dysfunction in neonates. Coordinating swallowing is not usually present before about 36 weeks of gestation. Also, the angle between the esophagus and stomach is less acute in infants than in adults, and the intrabdominal part of the esophagus is shorter. These anatomic differences lead to a higher rate of GER in children as compared to adults.

\section{Radiographic technique to evaluate the esophagus: The esophagram}

Esophagram is a radiographic imaging method using fluoroscopy and opaque CM. A marked reduction in a radiation dose has been achieved with an introduction of digital pulsed fluoroscopy and video fluoroscopy. The examination should be performed in lateral, antero-posterior, and oblique positions. The special equipment adapted for neonates and infants is highly recommended (Fig. 1). This equipment allows safer child immobilization, more accurate limitation of the field of view, and enables proper patient positioning. The examination is performed faster, and the radiation doze is significantly reduced.

Barium sulfate suspensions remain the $\mathrm{CM}$ of choice unless the esophageal perforation or the tracheoesophageal fistula (TEF) is suspected, and if there is a high probability of aspiration. When barium is contraindicated, low osmolar nonionic iodine CM (Iopami$\mathrm{dol} 61.24 \% \mathrm{w} / \mathrm{v}$ ) is preferred. Iopamidol produces a milder inflammatory response compared to barium sulphate suspensions in the case of aspiration, its effect on the blood gases is much lower, it evokes less edema and thus decreases the risk of a cardiovascular compromise. In neonates it is always safer to use Iopamidol (1, 3-5). 


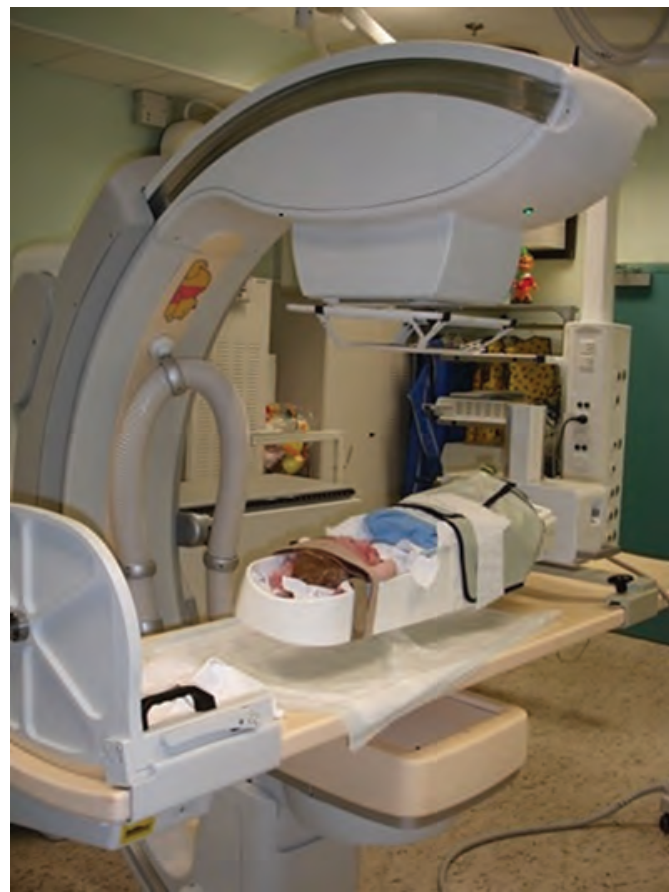

Fig. 1 Radiographic equipment ("mulda") adapted for the use in the newborn and infants up to $15 \mathrm{~kg}$.

Before starting the examination the radiologist should be familiar with the child's history of feeding and his or her clinical condition. Information about the child's appetite, changes in diet, ability to suck, the use of utensils, positioning, irritability and fatigue while fed, pain, signs of dysphagia, weight loss, respiratory symptoms (history of aspiration, coughing, choking while fed), changes in voice while crying and presence of GER should be collected. All this helps to determine the consistency and volume of feeding, utensils used, or specific foods that should be offered in order to decrease risks related to tracheobronchial aspiration. The protocol can be modified or shortened according to the child's clinical condition.

Therefore, team work is mandatory to perform the examination as safe as possible. Three phases should be evaluated during the esophagram (1) (Fig. 2 panel a).

In the first phase, termed the oral phase, food is received and prepared in the mouth.

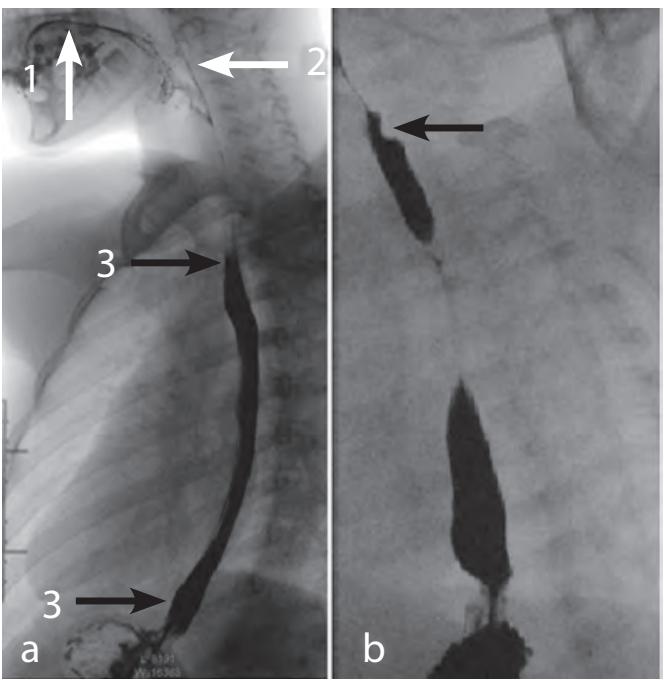

Fig. 2 The phases of esophagram: a) arrows point to anatomic regions, which are evaluated during the oral (1), pharyngeal (2) (white arrows), and esophageal phase (3) (black arrows); b) normal posterior impression on the lateral view (arrow) due to a contracted cricopharyngeus muscle.

The oral control of the bolus, tongue mobility and presence of residue in oral cavity are evaluated. Food is pushed into oropharynx, where the second phase, termed the pharyngeal phase, is initiated: the hypopharynx elevates, the epiglottis deflects, contraction of the pharynx begins and the cricopharyngeus muscle relaxes. In this phase velopharingeal motility (speech disorders) and closure (nasopharyngeal reflux of $\mathrm{CM}$ ), pulmonary aspiration (CM in tracheobronchial system), residue in vallecular or pyriphorm sinus, and cricopharyngeal incoordination are evaluated. In the third phase, termed the esophageal phase, the esophagus is evaluated from the cricopharyngeus to the LES. The esophagus should be well distended for optimal evaluation. The assessment of peristalsis and peristaltic waves is one of the main tasks in performing the esophagram. It is followed by the evaluation of the size of the esophageal lumen (stenosis, dilatation), esophageal con- 
tours, outside impressions, mucosa, position and function of GEJ.

In most children an UGI series is performed rather than an isolated esophagram. Therefore, the child is usually imaged from the oral cavity to the jejunum to also evaluate gastric emptying and duodenal loop fixation.

\section{Oral and pharingeal phase evaluation}

Swallowing is a complex process that requires integrity and coordination of many structures. When swallowing dysfunction is encountered, it is important to assess different types of food application and consistency. Often dysfunction may occur with certain types of application or consistency, but be alleviated when utilizing a different application or consistency. For instance, a child may aspirate when drinking thin liquid from a cup, but the aspiration may resolve when the child drinks nectar-thickened liquid from a bottle.

Difficulties related to sucking, chewing and other voluntary movements of the oral cavity are common in children with classic cerebral palsy (cortical motor deficits). An isolated pharyngeal phase dysfunction occurs in children with brainstem lesions and certain neurodegenerative conditions. Most patients, particularly those with diffuse hypoxic-ischemic injury, have a combined oral and pharyngeal phase dysphagia (6).

Nasopharyngeal reflux is the most common pathology found in this region, and may be caused by an abnormality of the neuromuscular mechanism which elevates the soft palate. As the name implies, esophagram will demonstrate reflux of CM into the nasopharynx (Fig. 3). The solution to this problem often involves a simple change in the food consistency.

The aspiration of CM in tracheobronchial system is a potentially dangerous, life threatening event for a child (Fig. 3). Therefore, the child's history related to aspiration episodes

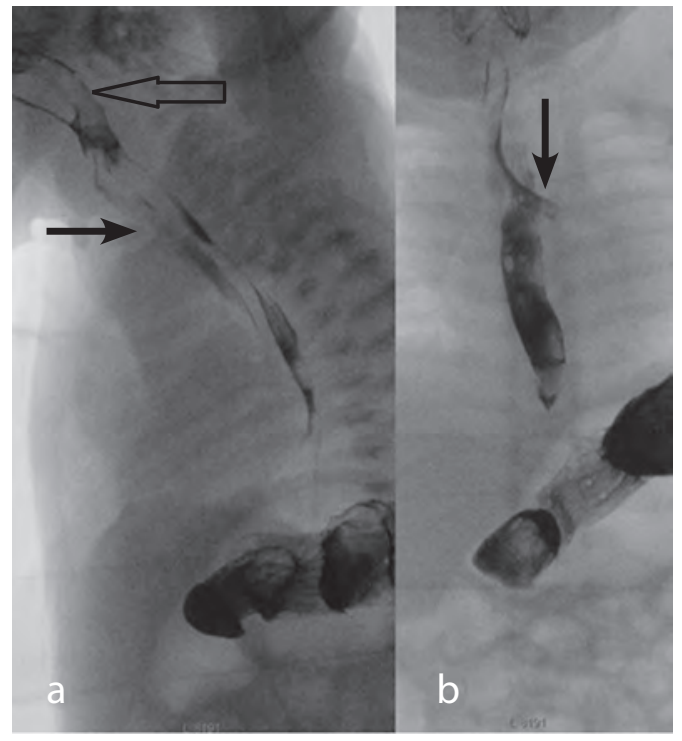

Fig. 3 Esophagram: a) the lateral view reveals nasopharyngeal reflux (arrow) and tracheobronchial aspiration (solid arrow) in a 1-month-old boy with recurrent aspiration pneumonias and feeding problems; b) aspiration in the left bronchus is clearly seen from the frontal view (black arrow).

is important and influences the choice of low osmolar nonionic iodine CM. Suction equipment should always be available in the examination room, in the event of aspiration. Aspiration is often caused by abnormal muscle coordination and/or function, which leads to defective coordination of the epiglottis and upper esophageal sphincter.

Cricopharynegal dysfunction should not be confused with normal cricopharyngeal impression, which is may be seen as a posterior impression caused by contracted cricopharyngeal muscle, when child is imaged in the lateral position. This is a normal variant in a patient without swallowing difficulties (Fig. 2 panel b). However, in symptomatic infants it could be abnormal $(3,6)$. Cricopharingeal dysfunction has been reported after trauma, in patients with autoimmune collagen disorders (scleroderma and dermatomyositis), and GER. 


\section{Esophageal phase evaluation}

The radiologist must evaluate several things during the esophageal phase. The first task is the evaluation of the peristalsis and different types of motility disorders. The evaluation of the esophageal lumen identifies esophageal stenosis, outside impressions, and mucosal abnormality (ulcers and erosions). The radiologist must be aware of esophageal congenital abnormalities which may be seen for the first time during esophagram. Finally, special focus should be paid to the GEJ: different types of hiatal hernia, the presence of GER and nonrelaxation of GEJ.

\section{Esophageal motility disorders}

Peristalsis is evaluated during real-time fluoroscopy. There are three types of peristaltic waves. The primary peristaltic wave is initiated by swallowing of a bolus of CM, and it is a distally progressive, lumen diminishing contraction wave. It propels the bolus through the esophagus. The secondary peristaltic wave follows the primary contraction and propels any remaining $\mathrm{CM}$ from the thoracic esophagus. It usually clears the esophagus of CM entirely or leaves only a small residual. Tertiary waves are nonpropulsive, uncoordinated esophageal contractions, not seen in healthy children (1) (Fig. 4 panel a).

Antiperistaltic waves in the lower part of the esophagus are often seen during the examination. They may be seen in many pathological conditions, and are not specific to any single disease. For instance, antiperistaltic waves are seen with motility disorders, GER, inflammation and after surgery.

Esophageal dysmotility is defined as a disruption of the primary peristalsis. It is also not specific to any one condition, and may be found in patients with neurologic disorders, connective tissue disorders and esophagitis.

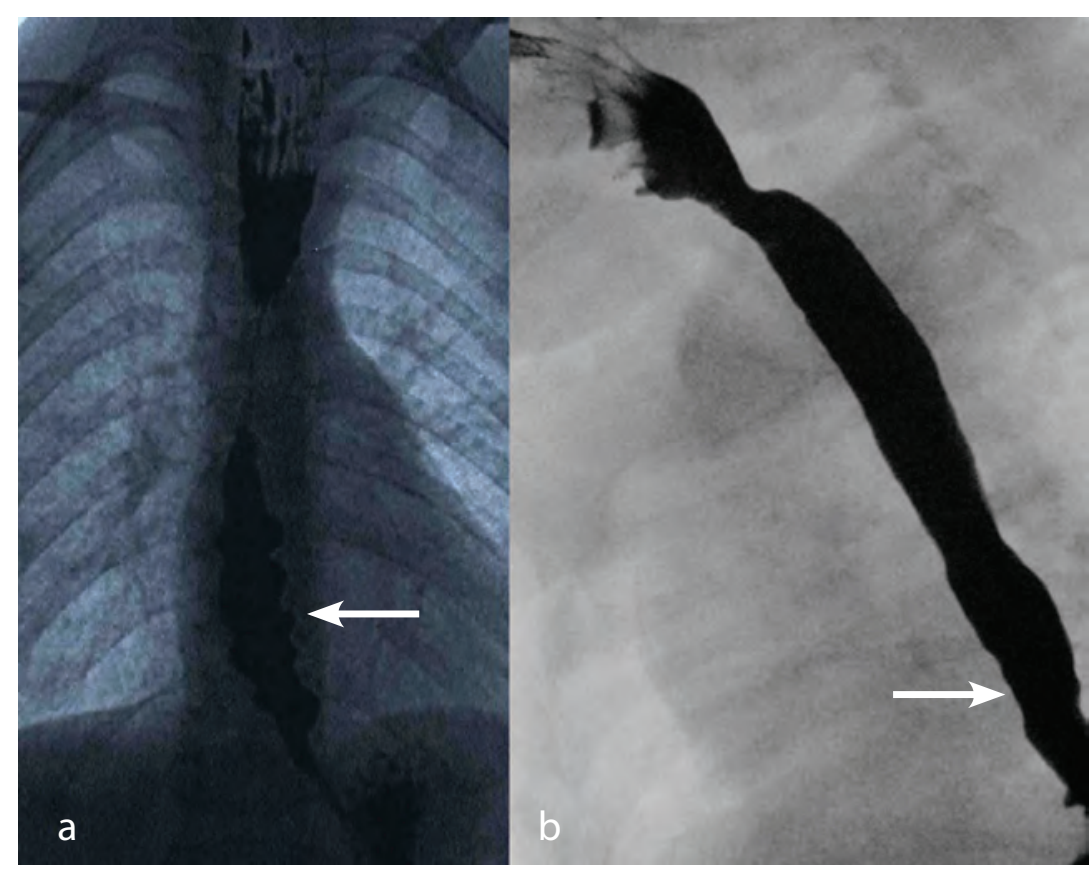

Fig. 4 Esophageal motility disorders: a) marked nonpropulsive tertiary contractions (arrow) in a 7.5-year-old boy with a triple A syndrome; b) atonia and aperistalsis with mild tertiary contractions (arrow) of the esophagus in a 1-month-old girl with swallowing difficulties. 
Patients with a history of the esophageal surgery frequently have abnormal peristalsis in the distal esophagus.

Hypoperistalsis or aperistalsis of the esophagus accompany neuromuscular disorders and may seriously affect the child's condition. The esophagus acts as a rigid (atonic) tube filled with CM, with or without tertiary contractions (Fig. 4 panel b). The risk of aspiration is very high. Disorders of the lower esophageal sphincter, including hypertonicity (achalasia) and over-relaxation (GER) are two other common motility disorders, which will be described in detail along with other GEJ disorders.

\section{Congenital esophageal malformations}

Congenital esophageal atresia with or without TEF is often diagnosed soon after birth when chest radiograph demonstrates a naso- gastric tube (NGT) coiled above the atretic portion of the esophagus, and esophagram is usually not needed. Esophagram is useful in the diagnosis of several other congenital lesions, such as isolated TEF (H-type or Ntype), esophageal duplication cysts, laryngotracheo-esophageal clefts (communications between the hypopharynx and larynx), and congenital esophageal stenosis $(3,7,8)$.

TEF is suspected in patients with unexplained chronic respiratory distress and recurrent pneumonia. The diagnosis of TEF can be challenging and frequently requires a combination of esophagram and endoscopy (bronchoscopy and esophagoscopy) (Fig. 5) (3, 7-10).

When TEF is suspected, a modification of the esophagram called "pull-back tube esophagram" is performed. During normal swallowing the esophagus may not be distended enough to allow the passage of $\mathrm{CM}$ into the

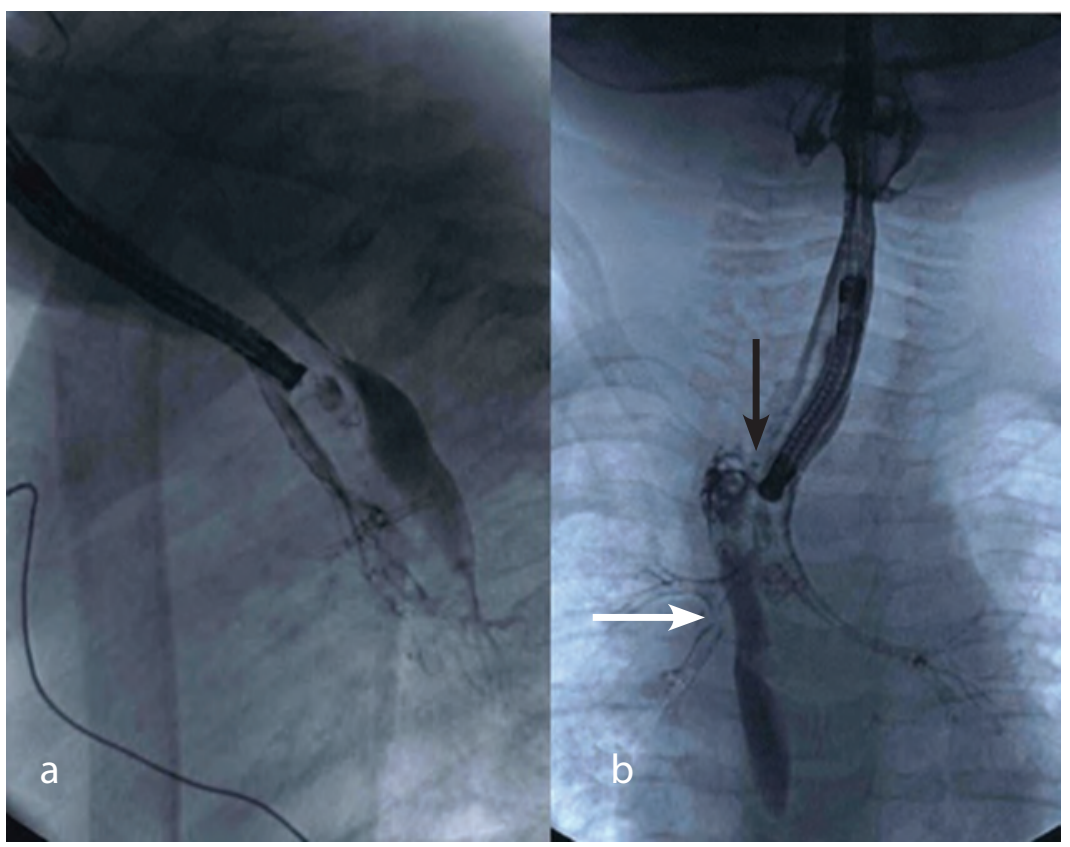

Fig. 5 Diagnostic imaging of the trachoesophageal fistula (TEF) in a) lateral; b) frontal view in an 8-month-old girl, who shows obstructive respiratory problems, coughing, and choking. She was operated on because of the esophageal atresia and TEF at birth. Bronchoscopy showed ligated fistula. In addition, another fistula was found below the ligated one and proved by injection of the contrast medium (black arrow). Stenosis of bronchus intermedius was also shown (white arrow) (33). 
fistula, which is often masked by redundant esophageal mucosa. In addition, the fistula typically runs cranially from the esophagus to the trachea. The NGT is inserted into the stomach. Under fluoroscopic guidance the NGT is pulled back into the lower third of the esophagus. 10 to $20 \mathrm{ml}$ of high-density water-soluble contrast is rapidly injected down the NGT to distend the lower part of the esophagus. During the procedure the child is screened in different positions. The NGT is then adjusted to lie in the mid and then the upper esophagus and the procedure are repeated. If there is no sign of TEF, a routine esophagram is performed. Some believe that pull-back tube esophagram has a limited role in the evaluation of the otherwise healthy child and should be reserved for patients who are intubated or who have associated abnormalities that significantly increase the risk of aspiration (11). The use of pre-operative CT scan in the evaluation of TEF is not accepted as a standard of care because of limited information obtained (12).

Esophageal duplication cysts usually do not cause symptoms and they are often discovered incidentally on chest radiograph. In some cases, they may cause dysphagia. Esophagram shows displacement of esophagus, and MR or CT should be performed in order to characterize these lesions $(8,13,14)$.

\section{Esophageal stenosis}

In the esophageal phase the caliber of the esophageal lumen is depicted and the radiologist evaluates for stenosis. A primary role of esophagram is to show the exact location, length and diameter of the stenosis, the presence of prestenotic dilatation, "rigidity" of the stenosis (how peristaltic waves pass through it), and the presence of an associated membranous web.

Congenital esophageal stenosis is a rare condition, resulting from a faulty embrio- genic separation of the respiratory and gastrointestinal system $(8,15)$. Esophagram typically shows a stenotic ring-shaped area on the distal third of the esophagus and a prestenotic dilatation (Fig. 6).

Esophagitis is relatively rare in otherwise healthy children, with the exception of children with GER. Infectious esophagitis is rare in healthy children, but occurs with a much higher frequency in immunocompromised children. Usually there is no need for imaging in patients with uncomplicated esophagitis. Esophagram is recommended in the evaluation of esophagitis complications,

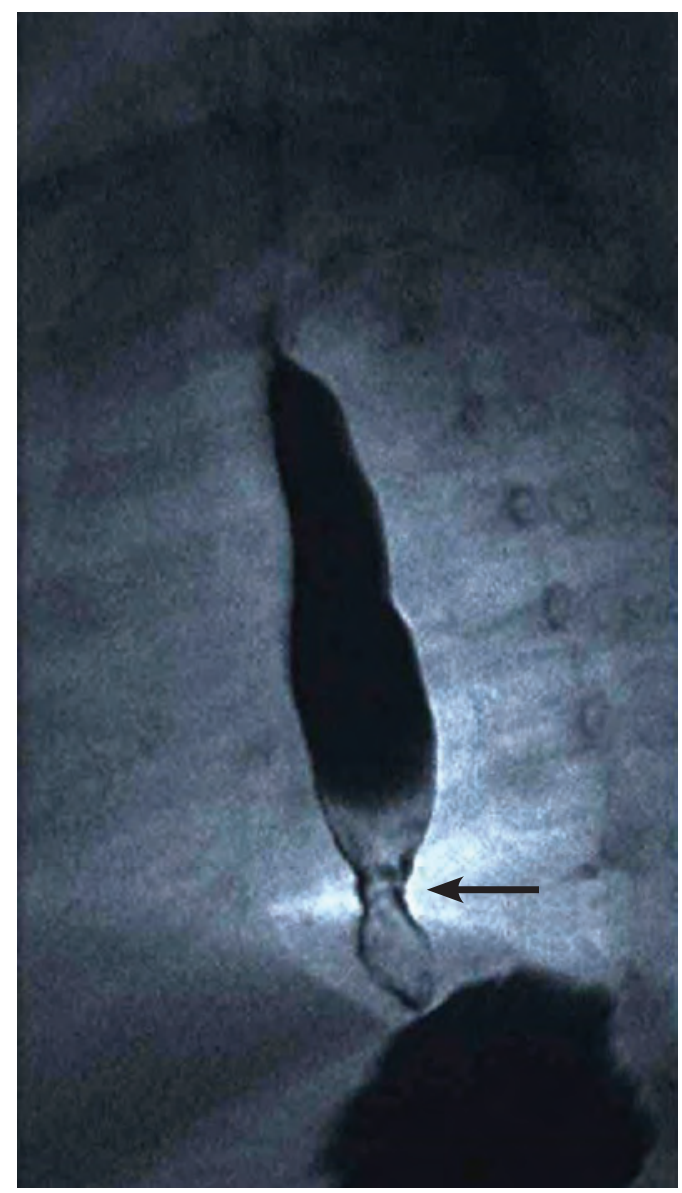

Fig. 6 Stenosis of the distal esophagus with a moderate proximal dilatation in a 1-year-old girl with dysphagia. Congenital stenosis was suggested by the location and shape of the narrowed segment. 
like the evaluation of strictures or stenosis of esophagus $(3,16)$. The etiology of the acquired esophageal stenosis is thus mainly post-inflammatory, mostly recognized as a complication of gastroesophageal reflux disease (GERD, Fig. 7), infection (Candida albicans, Fig. 8 panel a), cytomegalovirus, herpes simplex virus), it could be non-infective (epidermolysis bullosa dystrophica, Fig. 8 panel $b$, caustic ingestion), or related to various chronic diseases (Crohn's disease, chronic granulomatose disease, graft-versus-host disease, radiation therapy). Acquired stenosis can also be found after a surgical or endoscopical treatment (iatrogenic) and after trauma.
Esophagram has a limited role in the evaluation of suspected eosinophilic esophagitis. Nonspecific findings of GER and abnormal motility may be seen. Rarely, a ringed esophagus, a small caliber esophagus, and esophageal stricture can be seen. The esophagus appears normal on imaging in more than 50\% of pediatric patients with eosinophilic esophagitis. Therefore, in children with a typical clinical history of eosinophilic esophagitis, endoscopy and biopsy are indicated regardless of the findings on esophagram $(17,18)$.

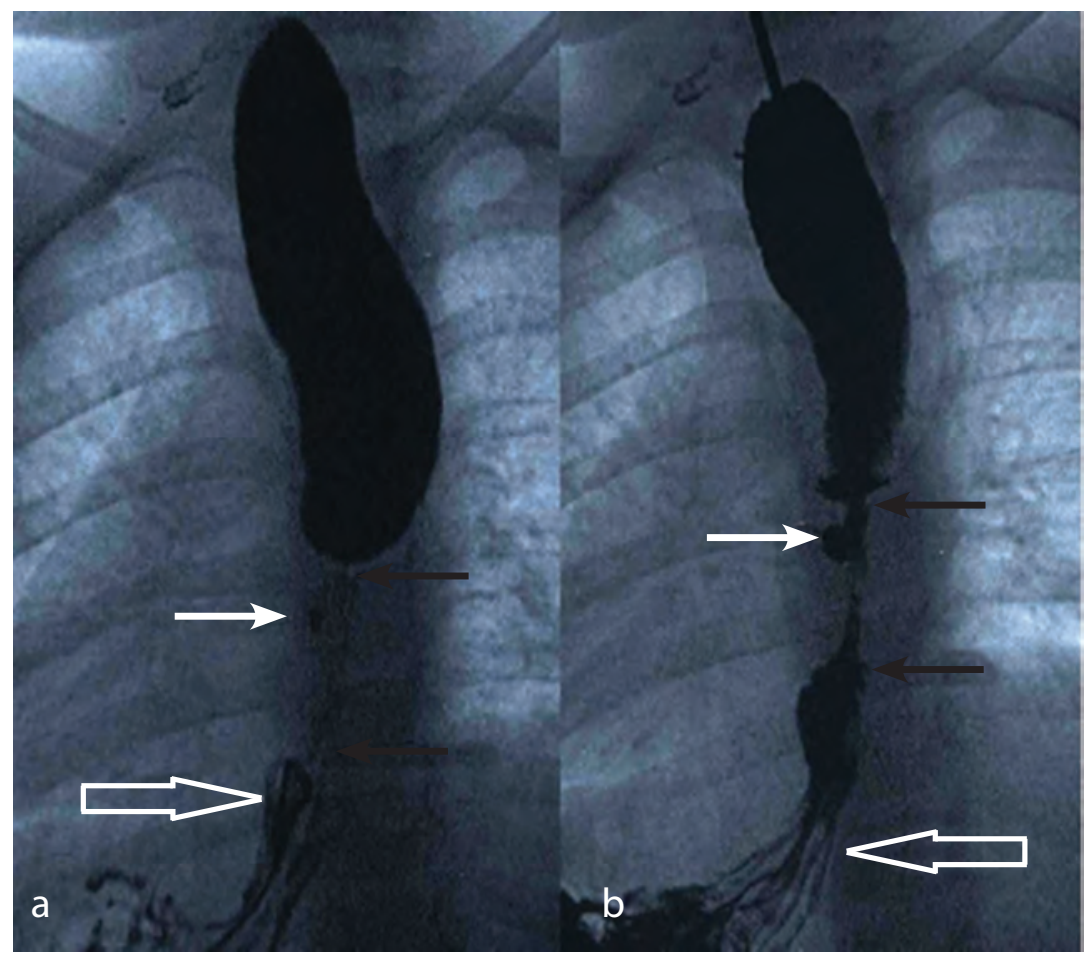

Fig. 7 Postinflammatory stenosis in a 2-year-old girl with a history of resistant gastroesophageal reflux and vomiting after every meal during the last month. Radiological signs of a gastroesophageal reflux disease: a) stenotic area in the distal esophagus is not filled with contrast medium (black solid arrow), a presence of prestenotic dilatation, irregular mucosa folds in the region of a hiatal hernia (white empty arrow); b) the length and width of the esophageal stenosis is clearly depicted (black solid arrow), diverticula are noted filled with a contrast medium (white solid arrow), hiatal hernia is present (black empty arrow). Motility disorder was observed during fluoroscopy. 

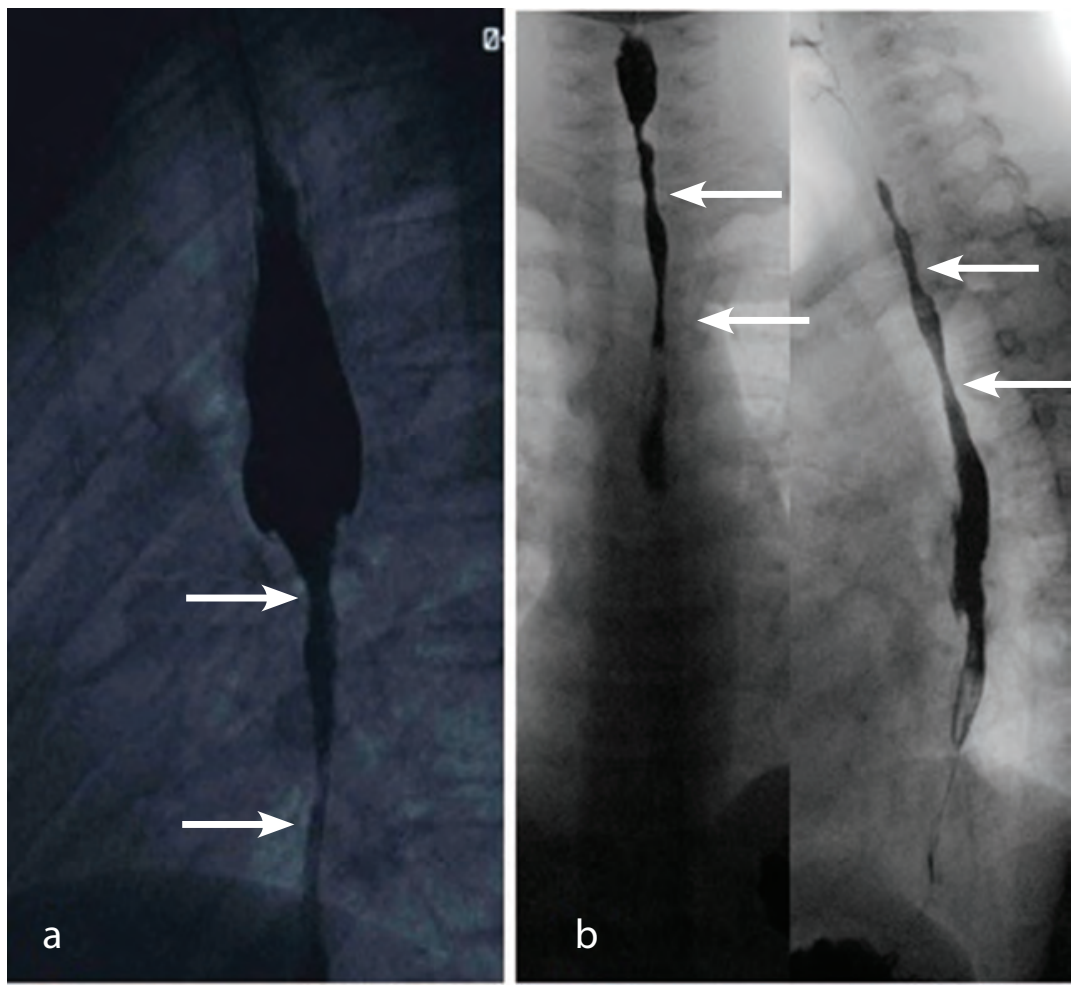

Fig. 8 Stenosis of the esophagus: a) Candida albicans esophagitis with a long stricture and prestenotic dilatation in a 16-year-old girl with K1 lymphoma; b) multiple stenoses of the esophagus in a 13-year-old boy with epidermolysis bullosa dystrophica.

\section{Disorders of GEJ}

The normal esophageal $\mathrm{B}$ ring, as described above, should not be confused with a Schatzki ring (a thin, circumferential submucosal ring that protrudes into the lumen of the distal esophagus), which is associated with dysphagia, hiatal hernia, eosinophilic esophagitis, and gastroesophageal reflux (GER). Whenever Shatzki ring is identified in children, endoscopy and biopsy should be considered $(19,20)$.

The most frequent disorders of the GEJ are GER, hiatal hernia with or without GER, paraesophageal hernia, and achalasia (2). The relationship between hiatal hernia, reflux and reflux esophagitis is controversial and poorly understood.

GER is physiologic in the first weeks and months of life. At that time it is difficult to differentiate pathologic GER from physiologic GER. Pathologic GER should be considered with progressively severe reflux after 6 weeks of age, lack of response to dietary and postural therapy, weight loss, failure to thrive, chronic respiratory symptoms, and/or bronchospasm $(21,22)$. The main purpose of esophagram in children with GER is to give anatomic and physiologic information, search for pathologic causes of GER (such as obstruction), and evaluate for aspiration. It also helps to evaluate for the complications of reflux esophagitis, such as motility disorders (dysmotility, tertiary contractions or aperistalsis), ulcerations, strictures and pseudodiverticula $(23,24)$. In cases of GER we always continue the contrast examination to the ligament of Trietz to exclude distal pathology, including obstruction of pyloric channel, duodenal stenosis and intestinal malrotation 

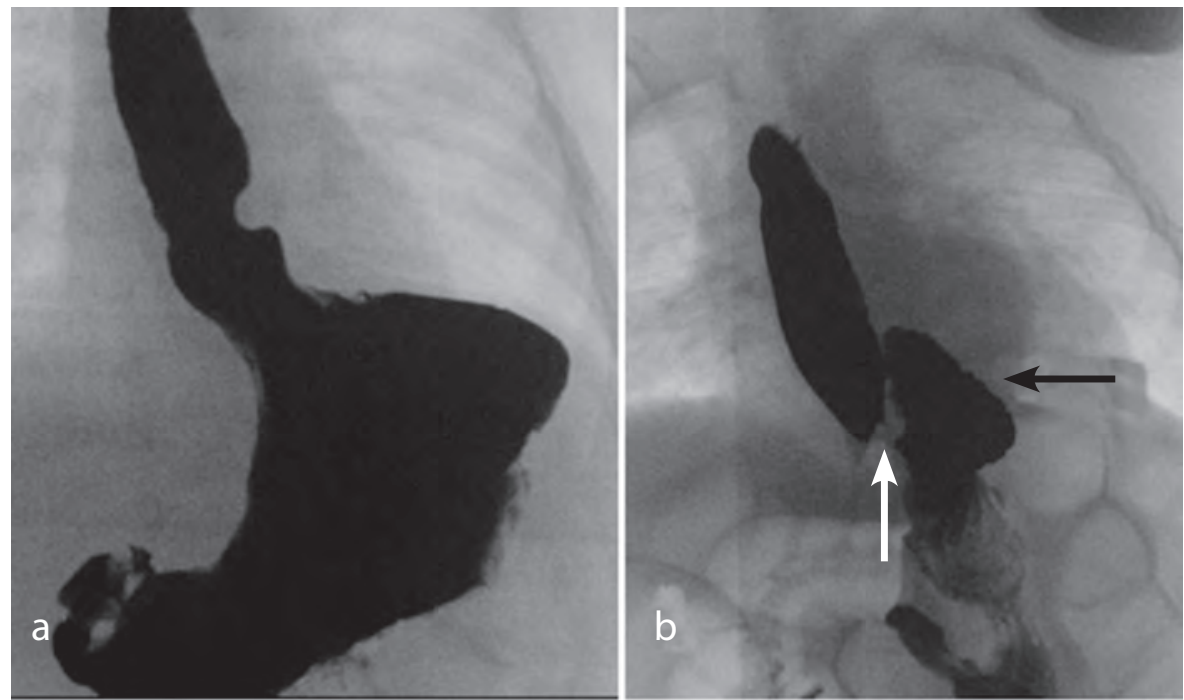

Fig. 9 Hiatal hernias: a) sliding hiatal hernia (gastroesophageal junction is above the hiatus) with gastroesophageal reflux; b) paraesophageal hiatal hernia with gastroesophageal junction under the diaphragm (white arrow) and a part of the stomach is herniated (black arrow) after fundoplication several years prior.

(5). US of GEJ can also be considered in the evaluation of GER (25).

In hiatal hernia the GEJ is situated in the thorax together with part of the stomach (Fig. 9 panel a). Sliding hiatal hernias, particularly if small, are often transient and clinically unimportant in the absence of GER. Larger hiatal hernias are uncommon and may be symptomatic. In paraesophageal hernia the GEJ remains intraabdominal, and a portion of the stomach herniates through the esophageal hiatus. It may be seen after fundoplication (Fig. 9 panel b).

Achalasia is defined as a failure of relaxation of the lower esophageal sphincter due to deficiency of cells in the Auerbach plexus. Children may complain of dysphagia, chest pain, and regurgitation of undigested food. On esophagram normal swallowing is seen with normal peristalsis to the level of aortic arch. Early in the course of the disease, uncoordinated peristalsis is found in the distal esophagus and the pathognomonic sign of lower esophageal "beaking" is seen (Fig. 10). In the advanced phase the entire esophagus may become atonic (26-28).

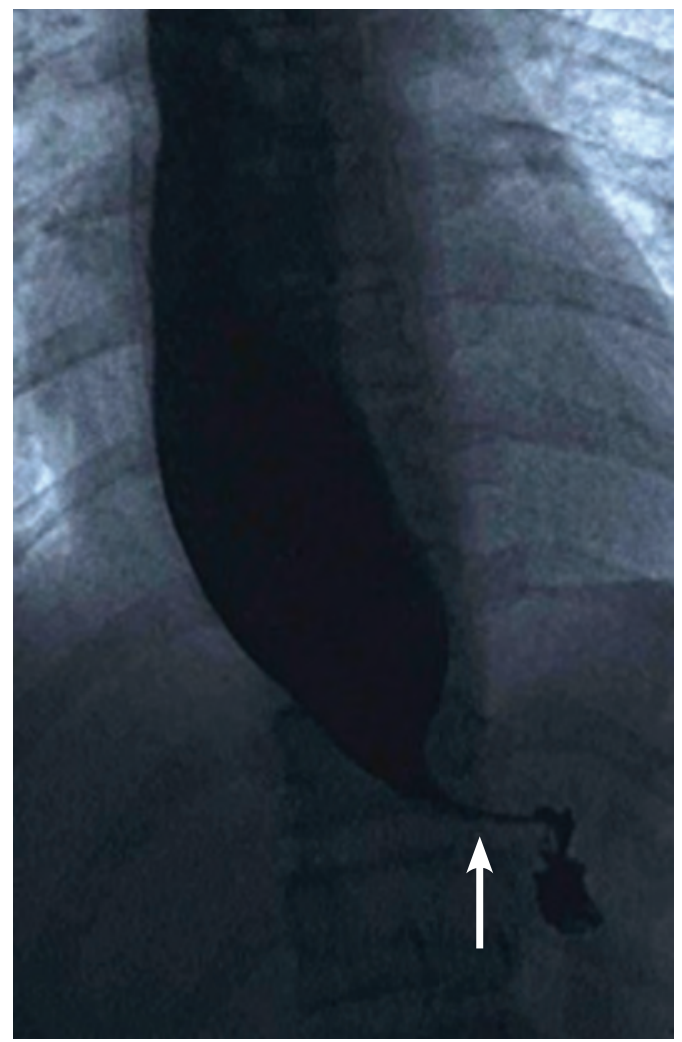

Fig. 10 Achalasia: dilatation of atonic esophagus and classic "beaking" of the gastroesophageal junction (white arrow) in a 11.5-year-old girl. 


\section{Outside impressions of esophagus}

The pathological outside indentations of esophagus are rare but important. It is crucial to differentiate between normal physiological and pathological esophageal impressions. Vascular rings, including the double aortic arch are the most common symptomatic conditions to cause pathological impressions. Other vascular rings include right aortic arch with aberrant left subclavian artery and left ligamentum arteriosum, and pulmonary sling. Less frequent pathologic outside impressions are caused by mediastinal masses. Obstructive symptoms caused by the compression of the airway (stridor, dyspnea during exertion, chronic cough, respiratory distress, reflex apnea and cyanosis) and the esophagus (dysphagia, recurrent vomiting, difficulty feeding and failure to thrive) are dominant clinical signs. Respiratory symptoms can worsen while child is fed. When vascular ring is suspected, esophagram is the first "screening" method to perform. A normal esophagram rules out a clinically important vascular ring. Classic S-shaped indentations in the frontal projection are highly suggestive of double aortic arches (Fig. 11).

A posterior vascular indentation may or may not be a vascular ring, but in the presence of a right aortic arch, a vascular ring should be suspected. A focal nodular indentation on

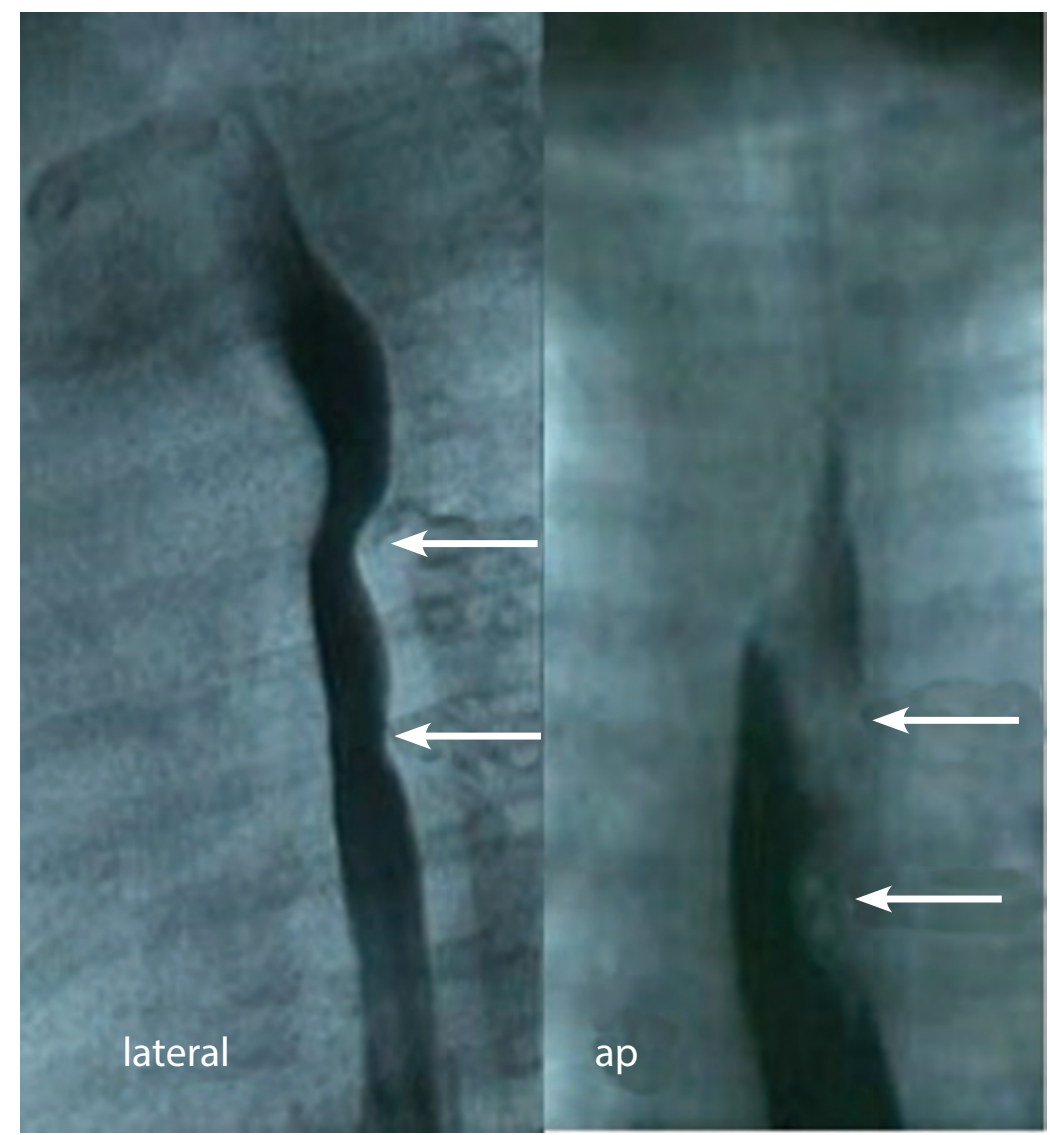

Fig. 11 Outside impressions of esophagus ina 5-month-old girl with a history of respiratory symptoms: a) lateral view reveals a posterior vascular impression; b) classic S-shaped impressions are seen from frontal view, which are highly suggestive of double aortic arch. 


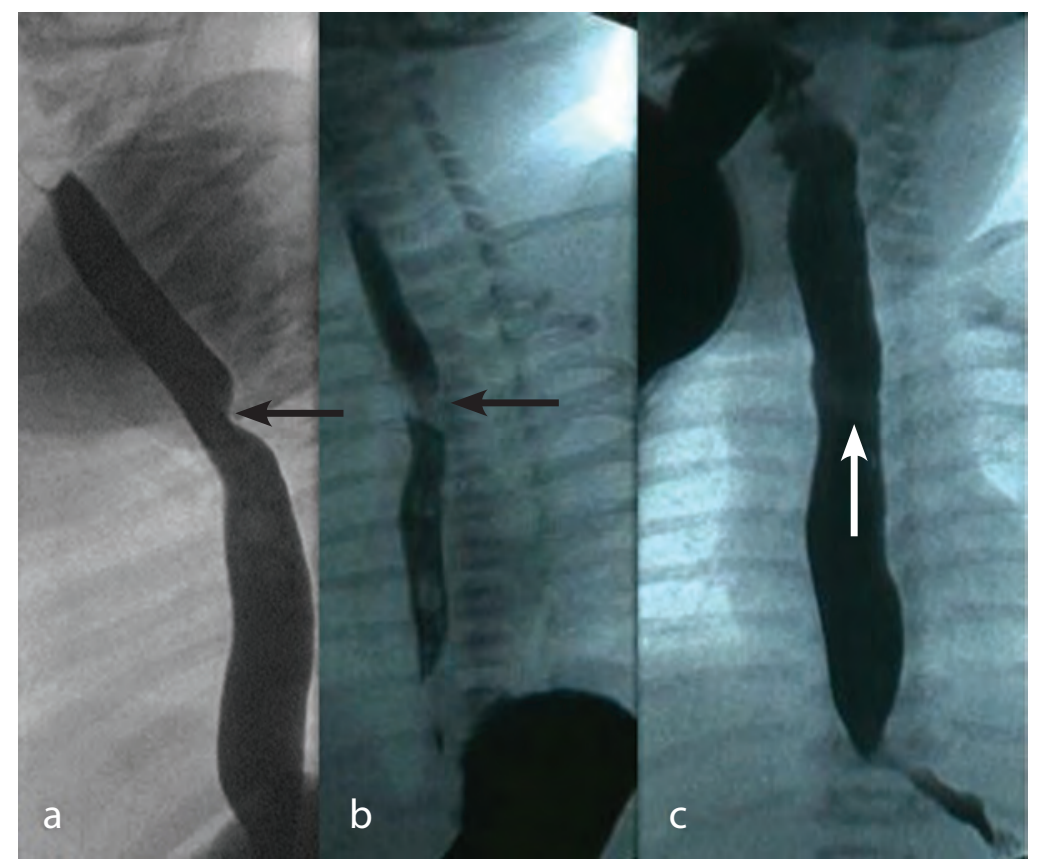

Fig. 12 Outside impression of the esophagus in a 7-day-old girl with a history of feeding problems. Oblique indentation of the esophagus is seen on a) lateral; b) oblique (black arrow); and c) frontal view (white arrow). Aberrant subclavian artery as a cause of feeding problems was suspected. Further imaging is obligatory to determine the type of the aberrant subclavian artery.

the anterior portion of the esophagus at the level of the carina seen on lateral esophagram is highly suspected for a pulmonary artery sling (29-32). Left-sided aortic arch with aberrant right subclavian artery is often found incidentally, but can rarely cause dysphagia. In this case the esophagus is indented posteriorly (Fig. 12). Definitive diagnosis requires contrast enhanced CT or MR angiography.

\section{Miscellaneous esophageal pathology}

Foreign bodies may be swallowed at any age. The symptoms of a foreign body in the esophagus may include drooling, inability to swallow solids, chest pain, and airway symptoms (stridor, wheezing, coughing). Radiopaque foreign bodies are easily detected on the plain chest radiograph and there is no need for further diagnostic imaging. Children with nonopaque esophageal foreign bodies (food, plastic, buttons, aluminum beverage can tabs) may require esophagram. Only a small amount of CM should be swallowed at the beginning of the examination because the degree of potential obstruction is not known. Esophageal foreign bodies often become lodged at normal areas of the relative narrowing, including at the aortic arch, above the GEJ or above strictures. The most dangerous foreign bodies are batteries, which could rapidly erode the esophageal mucosa and therefore require immediate treatment (3).

Esophageal trauma is rare. Most cases are iatrogenic, occuring during the placement of tubes and catheters, or during endoscopic or surgical procedures.

\section{Conclusion}

Radiographic imaging of swallowing and the esophagus remains the primary imaging 
modality in patients with a history of aspiration, dysphagia and feeding problems. It offers valuable diagnostic information related to anatomy, morphology and peristalsis. This relatively simple radiographic test is often sufficient to diagnose patient's underlying condition, and if not sufficient leads to suggestions for further imaging evaluation and treatment. Teamwork is mandatory to achieve the best possible diagnostic results. The radiologist must be familiar with the appearance of the pediatric esophagus in the normal state and be aware of the appearance in numerous pathological conditions in order to provide excellent patient care.

Conflict of interest: The authors declare that they have no conflict of interest.

\section{References}

1. Slovis TL. The normal esophagus. In Slovis TL and al. Caffey's Pediatric Diagnostic Imaging, 11th ed. Philadelphia: Mosby, 2008:2005-12.

2. Slovis TL. Disorders of gastroesophageal junction. In Slovis TL and al Caffey's Pediatric Diagnostic Imaging, 11th ed. Philadelphia: Mosby, 2008:2042-54.

3. Fordham LA. Imaging of the esophagus in children. Radiol Clin N Am. 2005;43(2):283-302.

4. McAlister W, Siegel MJ. Fatal aspirations in infancy during gastrointestinal series. Pediatr Radiol. 1984;14(2):81-3.

5. Helo N, Hoss D, Girguis M. Respiratory failure from an upper gastrointestinal series. Eur Respir Rev. 2014;23(133):396-7.

6. Slovis TL. Disorders of deglutition, peristalsis, and the velopharyngeal portal. In Slovis TL and al. Caffey's Pediatric Diagnostic Imaging, 11th ed. Philadelphia: Mosby, 2008:2021-6.

7. Hryhorczuk AL, Lee EY, Eisenberg RL. Esophageal abnormalities in pediatric patients. AJR. 2013;201(4):W519-32.

8. Slovis TL. Congenital esophageal malformations. In Slovis TL and al. Caffey's Pediatric Diagnostic Imaging, 11th ed. Philadelphia: Mosby, 2008:2013-20.
9. Ng J, Antao B, Bartram J, Raghavan A, Shawis R. Diagnostic difficulties in the management of H-type tracheoesophageal fistula. Acta Radiol. 2006;47(8):801-5.

10. Brookes JT, Smith MC, Smith RJ, Bauman NM, Manaligod JM, Sandler AD. H-type congenital tracheoesophageal fistula: University of Iowa experience 1985 to 2005. Ann Otol Rhinol Laryngol. 2007;116(5):363-8.

11. Laffan EE, Daneman A, Ein SH, Kerrigan D, Manson DE. Tracheoesophageal fistula without esophageal atresia: are pull-back tube esophagograms needed for diagnosis? Pediatr Radiol. 2006;36(11):1141-7.

12. Garge S, Rao KL, Bawa M. The role of preoperative CT scan in patients with tracheoesophageal fistula: a review. J Pediatr Surg. 2013;48(9):1966-71.

13. Hur J, Yoon CS, Kim MJ, Kim OH. Imaging features of gastrointestinal tract duplications in infants and children: from oesophagus to rectum. Pediatr Radiol. 2007;37(7):691-9.

14. Callahan MJ, Taylor GA. CT of the Pediatric Esophagus. AJR. 2003;181(11):1391-6.

15. Diab N, Daher P, Ghorayeb Z, Korkmaz G. Congenital esophageal stenosis. Eur J Pediatr Surg. 1999;9(3):177-81.

16. Slovis TL. Acquired esophageal lesions. In Slovis TL and al Caffey's Pediatric Diagnostic Imaging, 11th ed. Philadelphia: Mosby, 2008:2021-6.

17. Binkovitz LA, Lorenz EA, Di Lorenzo C, Kahwash S. Pediatric eosinophilic esophagitis: radiologic findings with pathologic correlation. Pediatr Radiol. 2010;40(5):714-9.

18. Diniz LO, Putnum PE, Towbin AJ. Fluoroscopic findings in pediatric eosinophilic esophagitis. Pediatr Radiol. 2012;42(6):721-7.

19. Buckley K, Buonomo C, Husain K, Nurko S. Schatzki ring in children and young adults: clinical and radiologic findings. Pediatr Radiol. 1998;28(11):884-6.

20. Towbin AJ, Diniz LO. Schatzki ring in pediatric and young adult patients. Pediatr Radiol. 2012;42:1437-40.

21. Fonkalsrud EW, Ament ME. Gastroesophageal reflux in childhood. Curr Probl Surg. 1996;33(1):1-70.

22. Lightdale JR, Gremse DA. Section on Gastroenterology, Hepatology, and Nutrition. Gastroesophageal reflux: management guidance for the pediatrician. Pediatrics. 2013;131(5):e1684-95. 
23. Vandenplas Y. Challenges in the diagnosis of gastroesophageal reflux disease in infants and children. Expert Opin Med Diagn. 2013;7(3):289-98.

24. Numanoglu A, Millar AJ, Brown RA, Rode H. Gastroesophageal reflux strictures in children, management and outcome. Pediatr Surg Int. 2005;21(8):631-4.

25. Savino A, Cecamore C, Matronola MF, Verrotti A, Mohn A, Chiarelli F, et al. US in the diagnosis of gastroesophageal reflux in children. Pediatr Radiol. 2012;42(5):515-24.

26. Pohl D, Tutuian R. Achalasia: an overview of diagnosis and treatment. J Gastrointestin Liver Dis. 2007;16(3):297-303.

27. Eckardt AJ, Eckardt VF. Current clinical approach to achalasia. World J Gastroenterol. 2009;15(32):3969-75.

28. Dughera L, Cassolino P, Cisarò F, Chiaverina M. Achalasia. Minerva Gastroenterol Dietol. 2008;54(3):277-85.
29. Kir M, Saylam GS, Karadas U, Yilmaz N, Çakmakçi $\mathrm{H}$, Uzuner N, et al. Vascular rings: presentation, imaging strategies, treatment, and outcome. Pediatr Cardiol. 2012;33(4):607-17.

30. Hernanz-Schulman M. Vascular rings: a practical approach to imaging diagnosis. Pediatr Radiol. 2005;35(10):961-79.

31. Yilmaz M, Ozkan M, Dogan R, Demircin M, Ersoy $\mathrm{U}$, Boke E, et al. Vascular anomalies causing tracheoesophageal compression: a 20-year experience in diagnosis and management. Heart Surg Forum. 2003;6(3):149-52.

32. Bonnard A, Auber F, Fourcade L, Marchac V, Emond S, Révillon Y. Vascular ring abnormalities: a retrospective study of 62 cases. J Pediatr Surg. 2003;38(4):539-43.

33. Kopriva S, Ključevšek D, Jereb S, Kenig T. Imaging of the tracheoesophageal fistula with fistulography (in Slovene). Endoskopska revija. 2002;7(18):95. 